\title{
Synthesis of Poly (2-aminothiazole) Using Chemical Oxidation Method
}

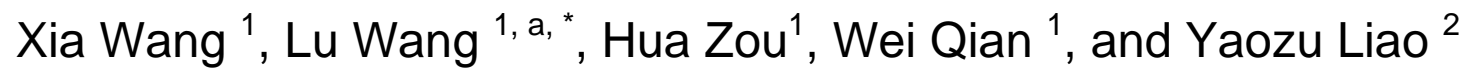 \\ ${ }^{1}$ School of Materials Science and Engineering, University of Shanghai for Science and Technology, \\ Shanghai, China \\ ${ }^{2}$ School of Chemistry, University of Bristol, Bristol, England BS8 1TS, UK, China \\ awanglu1390636@163.com
}

Keywords: Poly (2-aminothiazole), chemical oxidation, oxidation Method.

\begin{abstract}
Poly (2-aminothiazole) was synthesized by chemical oxidative polymerization of 2-aminothiazole, through selecting the appropriate reaction medium and simply modulating reaction parameters such as reaction time, temperature and oxidant concentrations, the chemical structure, product yield and the thermal stability of PAT are facilely optimized. Structural analysis of the polymer was carried out by FT-IR and UV-VIS spectroscopies. Yield was calculated using gravimetric method. Thermal properties were studied by TGA.
\end{abstract}

\section{Introduction}

Polymers with conjugated structure have attracted considerable interest in the past few decades. Because of their actual and potential applications in different technical fields, such as lithium ion battery, electro chromic materials, corrosion protection and sensors [1-4]. Some conventional polymers, such as polythiophene [5], polypyrrole [6] and polyaniline [4] have been extensively studied. Synthesis and characterization of novel conductive polymer material can enlarge the diversity with special properties.

Thiazole is a five-membered heteroaromatic compound, containing nitrogen, sulfur heteroatoms. It was favored by the majority of researchers because of its thermal stability and excellent conductivity properties, 2-aminothiazole (AT), since the introduction of the amino group, it had a lower oxidation potential and higher chemical reactivity, chemical oxidative polymerization is easier to conduct compared to thiazide. Due to its special chemical, electronic structure, AT can be involved in a variety of biochemical reactions, it has good functions such as bactericidal, anti-inflammatory, anti-viral, anti-corrosion, heavy metal ion adsorption and so on[7]. It can be anticipated, these superior performance of AT can be improved through functional group content increased after the polymerization. Apart from this, Poly (2-aminothiazole) (PAT) will has superior optical, electrical properties with the formation of a large $\pi$-conjugated structure. So PAT will become a kind of new organic polymer material transcend traditional conductive polymers.

PAT can be synthesized by chemical and electrochemical method [8-9]. The chemical polymerization technique is very useful for obtaining large amounts of the polymer, while the electrochemical synthesis technique is more suitable for gaining smooth polymers. It has been reported dehydrogenation synthesis of poly (2-aminothiazole) by the electrochemical oxidation polymerization method. However, the product is entirely dependent on the electrode area and the yield is low so that large-scale synthesis is difficult. Compared with the electrochemical oxidation, chemical oxidation method is simple, mild, high yield, low energy consumption, easy to promote mass production, more effective to avoiding the shortcomings of electrochemical polymerization.

\section{Experimental}

AT monomer was dissolved in $10 \mathrm{ml}$ solvents. $\mathrm{FeCl}_{3}$ as an oxidant was dissolved in corresponding solvents. The amount of the $\mathrm{FeCl}_{3}$ was added drop wise to the monomer solution. Control the reaction temperature, the reaction was stirred for a certain time. The resulting product was filtered, washed, and dried. 
FT-IR spectra were recorded on a Spectrum 100 FT-IR spectrometer using $\mathrm{KBr}$ pellets. Absorption spectra were measured using a Lambda $750 \mathrm{UV}$-vis spectrophotometer in DMSO. Thermogravimetric analyses (TGA) of the samples were conducted in $\mathrm{N}_{2}$ at a heating rate of $10{ }^{\circ} \mathrm{C} / \mathrm{min}$ from room temperature to $600{ }^{\circ} \mathrm{C}$ using a Perkin-Elmer Pyris 1 TGA apparatus.

\section{Results and Discussion}

Several representative oxidant species potentials (ORPs) was reported to be selected to optimize the polymerization with different standard oxidation reduction, including $\mathrm{NaClO}(1.63 \mathrm{~V}), \mathrm{H}_{2} \mathrm{O}_{2}(1.77 \mathrm{~V})$, $\mathrm{H}_{2} \mathrm{O}_{2} / \mathrm{FeCl}_{2}(2.80 \mathrm{~V}),\left(\mathrm{NH}_{4}\right)_{2} \mathrm{~S}_{2} \mathrm{O}_{8}(2.01 \mathrm{~V}), \mathrm{FeCl}_{3}(0.77 \mathrm{~V})$.

This article select oxidant with minimum ORPs for exploratory. In order to select a suitable solvent serves as the reaction medium, the solubility of the monomers and oxidant in different solvents were test. In methanol, ethanol, acetone, nitromethane, both monomer and oxidant can dissolve simultaneously. It means that with the help of these solvents, contacting of oxidants and monomer in the molecular level can be achieved to ensure that the reaction proceed sufficiently, so that these solvents meet the necessary conditions of the reaction medium.

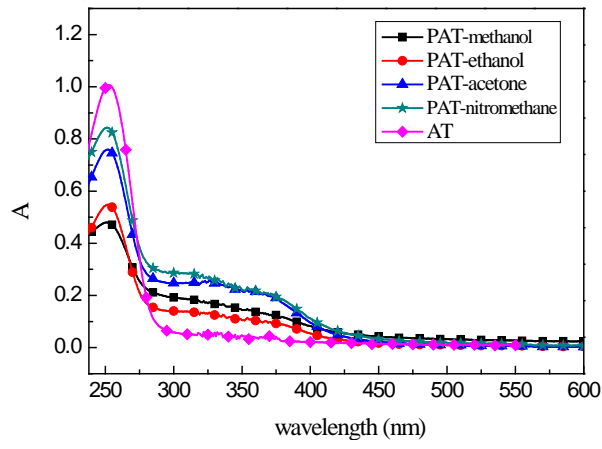

Fig.1. UV-VIS absorption spectra of AT and PAT in different solvent

The UV-VIS absorption spectra of PAT dissolved in DMSO displayed the formation of conjugated structure in Figure1.The peak at $265 \mathrm{~nm}$ could be ascribed to the $\pi \rightarrow \pi^{*}$ transition of thiazide ring of 2AT. The broad absorption bands at $350 \mathrm{~nm}$ with a long tail extending to $500 \mathrm{~nm}$ could be attributed to $\pi \rightarrow \pi^{*}$ transition of the conjugated polymers.

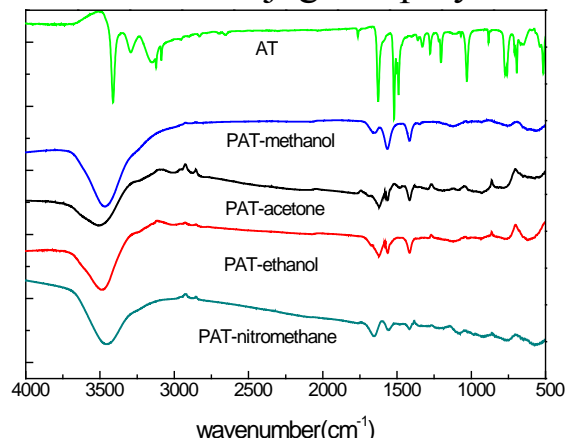

Fig.2. FT-IR spectra of AT and PAT reaction in different solvent

The FT-IR spectra of the 2AT monomer and PAT are shown in Figure 2. the bands at $3414 \mathrm{~cm}^{-1}$ and $3297 \mathrm{~cm}^{-1}$ are attributed to the asymmetric and symmetric $\mathrm{N}-\mathrm{H}$ stretching of the $-\mathrm{NH}_{2}$ group in AT, respectively. These two bands turned to a single broad band at 3476, 3502, 3486, 3468 $\mathrm{cm}^{-1}$ in methanol, acetone, ethanol, and nitromethane respectively, assigned to $\mathrm{N}-\mathrm{H}$ stretching. It indicated the oxidation of $-\mathrm{NH}_{2}$ and formation of $-\mathrm{N}(\mathrm{H})$ - linkages between the 2AT rings.

System color and products shape changed during the reaction as shown in Table1. In nitromethane, the amounts of the product is more and the color is darker, it also intensely precipitate in the bottom of the bottle. This shows that it is more beneficial to polymerization and separate when nitromethane is the solvent.

The reaction time and temperature are two important parameters for the synthesis of PAT, they closely related to the performance of the reaction product. In general, as the reaction temperature increased, the reaction time will be shortened, but it is not conducive to the improvement of the 
molecular weight and distribution. However, reducing the temperature is not beneficial to improve the yield of the polymerization, and if the reaction time was too long, the molecular chain has been formed will be degraded in the oxidizing environment. In addition, the amount of the oxidant also affect the progress of the reaction.

Table 1 Reaction phenomenon

\begin{tabular}{cccc}
\hline solvent & Color of AT & Color of PAT & Feature of products \\
\hline methanol & faint yellow & red & small amount of black solid \\
ethanol & faint yellow & red brown & small amount of black precipitate \\
acetone & faint yellow & brownness & large number of black floccules \\
nitromethane & faint yellow & puce & large number of black solid precipitation \\
\hline
\end{tabular}

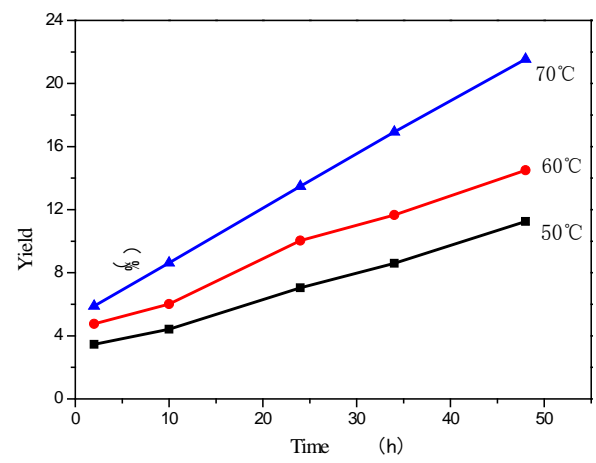

Fig.3. effect of reaction time and temperature on the yield of PAT

In this section, the yield of polymerization as shown in Figure 3, with the increasing of reaction time and temperature, the yield was increased. It suggested that, a suitable increasing of the reaction temperature and the time will be beneficial to the synthesis of the polymer. Considering the safety of solvent in high temperature and description of Figure 3, the study extended reaction times when the reaction condition is $70^{\circ} \mathrm{C}$.

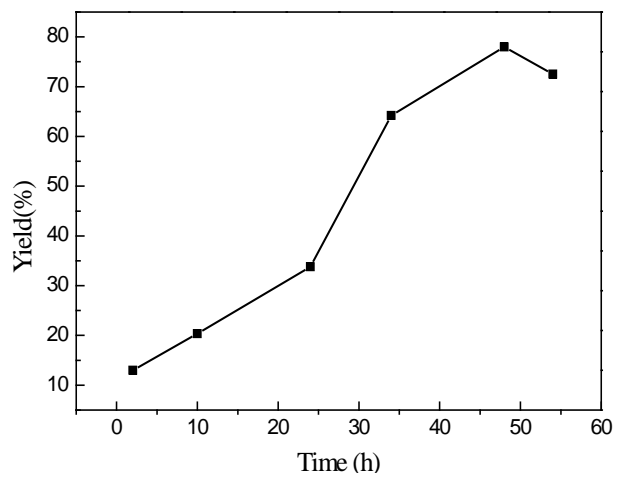

Fig.4 Effect of reaction time on the yield of PAT

Figure 4 shows the presence of automatic acceleration process. It is due to that the hindered the movement of long. However, monomer is relatively small, so the increase of the viscosity cannot hinder polymerization of the monomers. As the reaction continues, the presence of excess oxidant, and the oxygen in the air will cause over-oxidation, the long-chain structure is destroyed, cracked into smaller molecular, polymers will dissolve in the solvent and lost during filtration when the temperature is high.

Thermal stability of the product has a great influence on the application. As shown in figure 5 and figure 6 , relative to monomers, the thermal stability of polymers have significantly improved, and char yield are greatly enhanced. Furthermore, initial decomposition temperature was $282^{\circ} \mathrm{C}$ at $24 \mathrm{~h}$,it improved to $306{ }^{\circ} \mathrm{C}$ at $48 \mathrm{~h}$, it indicated that with the increasing of reaction time, the thermal stability of the polymer getting better ( Fig.5). 


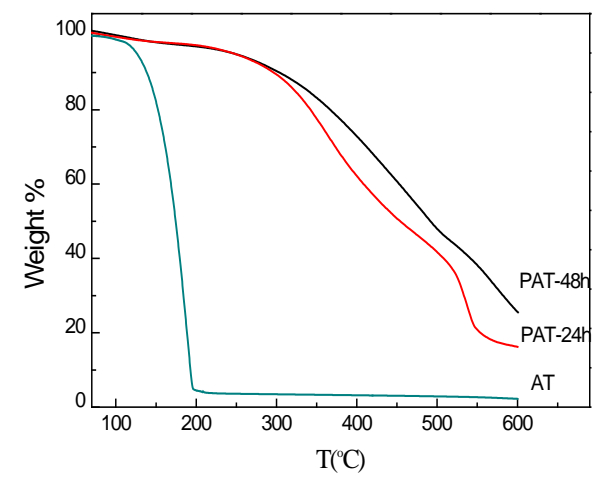

Fig.5 TG curves of PAT at different time

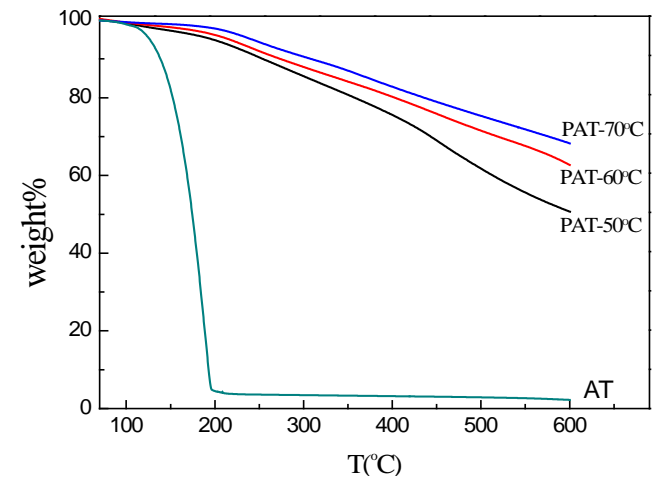

Fig.6 TG curves of PAT at different temperature

Initial decomposition temperature was $161^{\circ} \mathrm{C}, 178^{\circ} \mathrm{C}, 199^{\circ} \mathrm{C}$, and the char yield was $50.7 \%, 62.7 \%$, $68.3 \%$ respectively when the reaction temperature was $50^{\circ} \mathrm{C}, 60^{\circ} \mathrm{C}, 70^{\circ} \mathrm{C}$ (Fig.7). This suggests that increasing the reaction temperature is also conducive to improve the thermal stability of the product effectively.

Thus, through the rational design of experiments, the appropriate reaction conditions will severely affect the performance and efficiency of the polymeric products.

\section{Conclusions}

In summary, in this paper, PAT were successfully synthesized by chemical oxidative polymerization method using the FeCI3 dissolved in nitromethane. The structures of the polymers were clarified by FT-IR, UV-VIS, when the reaction condition was $70^{\circ} \mathrm{C}, 48 \mathrm{~h}$, the yield and thermal stability shows satisfactory results.

\section{Acknowledgements}

This work is supported by the National Natural Science Foundation of China (51203090, Y.Z.L.), the Natural Science Foundation of Shanghai (12ZR1446700, Y.Z.L.), the Innovation Program of Shanghai Municipal Education Commission (13YZ074, Y.Z.L.) and the Hujiang Foundation of China (B14006, H.Z.).

\section{References}

[1] Odaci D, Kayaham SK, Timur S, Toppare L. Electrochim Acta 53: p.4104-4108 (2008).

[2] Retama JR, Mecerreyes D, Lopez-Ruiz B. Colloids Surf a 270: p.239-244 (2005)

[3] Armelin E, Pla R, Liesa F, Ramis X, Iribarren JI, Aleman C. Corros Sci 50: p.721-728 (2008)

[4] Rehan HH. J Power Sources 113: p.57-61 (2003)

[5] Kabasakaloglu M, Talu M. Appl Surf Sci 218: p.84-96(2003)

[6] Ahuja T, Mir IA, Kumar D, Rajesh D. Sensors Actuators B 134: p.140-145(2008)

[7] Zhou X, Shao L, Jin Z, Liu JB, Dai H, Fang JX. Heteroat Chem 18(1): p.55-59 (2007)

[8] Ciftci H, Testereci HN, Oktem Z. Polym Bull 66: p.747-760 (2011)

[9] Yıldırım M, Kaya I. Synth Met 162: p.436-443 (2012). 